\title{
A MEASURE OF NON-IMMERSABILITY OF THE GRASSMANN MANIFOLDS IN SOME EUCLIDEAN SPACES
}

\author{
by CORNEL PINTEA
}

(Received 19th April 1996)

Let $G_{k, n}$ be the Grassmann manifold consisting in all non-oriented $k$-dimensional vector subspaces of the space $\mathbf{R}^{k+n}$. In this paper we will show that any differentiable mapping $f: G_{k, n} \rightarrow \mathbf{R}^{m}$, has infinitely many critical points for suitable choices of the numbers $m, n, k$.

1994 Mathematics subject classification: 57R70.

\section{Introduction}

Recall that $G_{k, n}$ is a compact manifold of dimension $k n$ and that the manifold $G_{1, n}$ is just the real projective space $P_{n}(\mathbf{R})$.

In the paper [4] it is proved that the Grassmann manifolds $G_{2, n}$ and $G_{2, s-1}$, where $s=2^{r}$ is such that $2^{r-1} \leq n<2^{r}$, cannot be immersed in the euclidean spaces $\mathbf{R}^{2 s-3}$ and $\mathbf{R}^{3 s-3}$ respectively. This means that any differentiable mapping $f: G_{2, n} \rightarrow \mathbf{R}^{2 s-3}$ or $g: G_{2, s-1} \rightarrow \mathbf{R}^{3 s-3}$, has one critical point at least. This observation justifies the investigations on the cardinal number

$$
\varphi(M, N)=\min \left\{|C(f)|: f \in C^{\infty}(M, N)\right\}
$$

called the $\varphi$-category of the pair $(M, N)$ of the differentiable manifolds $M$ and $N$. The $\varphi$-category of the pair $(M, N)$ represents a measure of non-immersability of the manifold $M$ into the manifold $N$ if $\operatorname{dim} M<\operatorname{dim} N$, and it is a measure of the distance of the pair $(M, N)$ from a fibration of the manifold $M$ over $N$, if $\operatorname{dim} M \geq \operatorname{dim} N$ and $M, N$ are compact manifolds. If $|C(f)|$ is infinite for all $f \in C^{\infty}(M, N)$, we shall use the notation $\varphi(M, N)=\infty$. In the present paper the $\varphi$-category of the pairs $\left(G_{2, n}, \mathbf{R}^{m}\right)$, $\left(G_{3, n}, \mathbf{R}^{m}\right)$ and $\left(P_{n}(\mathbf{R}), \mathbf{R}^{m}\right)$ will be studied.

\section{Preliminary results}

The following theorem is the principal result of the paper.

Theorem 2.1. Let $M^{m}, N^{n}$ be smooth manifolds such that $m<n$ and $f: M \rightarrow N$ be 
an immersion. If $y \in \operatorname{Im} f$ is such that $f^{-1}(y)$ is finite, then there exists an immersion $g: M \rightarrow N \backslash\{y\}$

Proof. Supposing that $f^{-1}(y)=\left\{x_{1}, \ldots, x_{p}\right\}$, there exists the local charts $\left(U_{i}, \varphi_{i}\right)$, $\left(V_{i}, \psi_{i}\right), i \in\{1,2, \ldots, p\}$ and the real positive number $r$, such that

(i) $\bar{U}_{i} \cap \bar{U}_{j}=\emptyset$ for $i \neq j$;

(ii) $y \in \bigcap_{i=1}^{p} V_{i}, x_{i} \in U_{i}, \varphi\left(x_{i}\right)=0, \psi_{i}(y)=0(\forall) i \in\{1,2, \ldots, p\}$;

(iii) If $D_{\varphi}^{s}$ denotes the pre-image of the open disk $D=\left\{x \in \mathbf{R}^{k} \mid\|x\|<s\right\}(k \in\{m, n\})$ by a coordinate mapping $\varphi: U \rightarrow \mathbf{R}^{k}$ with $\varphi(0)=0$ and $D \subseteq \varphi(U)$, then $\bar{D}_{\varphi_{1}}^{2 r} \subseteq U_{i}$ and $\bar{D}_{\varphi_{i}}^{2 r} \subseteq \bigcap_{i=1}^{p} V_{i},(\forall) i \in\{1,2, \ldots, p\}$;

(iv) $\left(\psi_{i} \circ f \circ \varphi_{i}^{-1}\right)\left(x_{1}, \ldots, x_{m}\right)=(x_{1}, \ldots, x_{m}, \underbrace{0, \ldots, 0}_{n-m \text { times }})(V) i \in\{1,2, \ldots, p\}$.

Consider the smooth positive functions $\theta_{i}: N \rightarrow \mathbf{R}$ which has the properties $\theta_{i}^{-1}(0)=N \backslash D_{\varphi_{1}}^{r}$ and the smooth vector fields $X_{1}, X_{2}, \ldots, X_{p}$ which are defined on $N$ by

$$
X_{i}(z)= \begin{cases}\left.\theta_{i}(z) \frac{z^{\psi_{i}}}{\partial x_{n}}\right|_{z} & \text { if } z \in D_{\psi_{i}}^{2 r}, \\ 0 & \text { if } N \backslash D_{\psi_{i}}^{r}\end{cases}
$$

Obviously the norms $\left\|X_{1}\right\|, \ldots,\left\|X_{p}\right\|$ of the fields $X_{1}, X_{2}, \ldots, X_{p}$ are bounded with respect to any Riemannian metric on $N$, namely they are completely integrable (see [5, pp. 183]). Denote by $\alpha_{i}^{i}$ the global flow induced by $X_{i}$ and consider the projection $\beta: \mathbf{R}^{n} \rightarrow \mathbf{R}, \beta\left(x_{1}, \ldots, x_{n}\right)=x_{n}$. Observe that

$$
\left(\beta \circ \psi_{i} \circ f \circ \varphi_{i}^{-1}\right)\left(x_{1}, \ldots, x_{m}\right)=0(\forall) x=\left(x_{1}, \ldots, x_{m}\right) \in \varphi_{i}\left(U_{i}\right) .
$$

One can therefore say that

$$
\left(\beta \circ \psi_{i} \circ f\right)(x)=0(\forall) x \in D_{\varphi_{i}}^{2 r} .
$$

Define the mapping $g$ in the following way:

$$
g(x)=\left\{\begin{array}{cc}
\alpha_{1}^{1}(f(x)) & \text { if } x \in D_{\varphi_{1}}^{2 r} \\
\vdots & \vdots \\
\alpha_{1}^{p}(f(x)) & \text { if } x \in D_{\varphi_{p}}^{2 r} \\
f(x) & \text { if } x \in M \backslash \bigcup_{i=1}^{p} D_{\varphi_{1}}^{r}
\end{array}\right.
$$

Because $\alpha_{2}^{1}, \ldots, \alpha_{p}^{1}$ are diffeomorphisms and $f$ is an immersion, it follows that $g$ is also an immersion. It remains only to show that $y \notin \operatorname{Img}$, that is, $\beta\left(\psi_{i}(g(x))\right)>0(V) x \in D_{\phi_{1}}^{2 r}$ and $(\forall) i \in\{1,2, \ldots, p\}$. Further on, we have successively 


$$
\begin{aligned}
\frac{d}{d t}\left[\psi_{i}\left(\alpha_{i}^{i}(y)\right)\right] & =\left(d \psi_{i}\right)_{\alpha_{i}^{\prime}(y)}\left(\frac{d}{d t} \alpha_{i}^{i}(y)\right)=\left(d \psi_{i}\right)_{\alpha_{i}^{\prime}(y)}\left(X_{i}\left(\alpha_{i}^{i}(y)\right)\right)=\left(d \psi_{i}\right)_{\alpha_{i}^{\prime}(y)}\left(\left.\theta_{i}\left(\alpha_{t}^{i}(y)\right) \frac{\partial^{\psi_{i}}}{\partial x_{n}}\right|_{\alpha_{i}^{j}(y)}\right) \\
& =\theta_{i}\left(\alpha_{t}^{i}(y)\right)\left(d \psi_{i}\right)_{\alpha_{i}^{i}(x)}\left(\left.\frac{\partial^{\psi_{i}}}{\partial x_{n}}\right|_{\alpha_{i}^{i}(y)}\right)=\theta_{i}\left(\alpha_{t}^{i}(y)\right) e_{n}=\left(0, \ldots, 0, \theta_{i}\left(\alpha_{l}^{i}(y)\right)\right) .
\end{aligned}
$$

Hence for $x \in D_{\varphi_{1}}^{r}$ we have

$$
\beta\left(\psi_{i}(g(x))\right)=\beta\left(\psi_{i}\left(\alpha_{1}^{i}(f(x))\right)\right)=\int_{0}^{1} \theta_{i}\left(\alpha_{s}^{i}(f(x))\right) d s>0 .
$$

Remark The mapping $g$ constructed above is homotopic to $f$ relative to the set $M \backslash \bigcup_{i=1}^{k} D_{\varphi_{i}}^{r}$. More precisely we have the relation

$$
f \simeq_{H} g\left(\operatorname{rel} M \backslash \bigcup_{i=1}^{k} D_{\varphi_{i}}^{r}\right)
$$

where $H:[0,1] \times M \rightarrow N$ is given by

$$
H(t, x)=\left\{\begin{array}{cc}
\alpha_{\imath}^{1}(f(x)) & \text { if } x \in D_{\varphi_{1}}^{2 r} \\
\vdots & \vdots \\
\alpha_{t}^{p}(f(x)) & \text { if } x \in D_{\varphi_{k}}^{2 r} \\
f(x) & \text { if } x \in M \backslash \bigcup_{i=1}^{p} D_{\varphi_{k}}^{r} .
\end{array}\right.
$$

Corollary 2.2. Let $M^{m}, N^{n}$ be smooth manifolds such that $M$ is compact and $m<n$. If $f: M \rightarrow N$ is an immersion and $y_{1}, \ldots, y_{1} \in N$ are values of $f$, then there exists an immersion $g: M \rightarrow N \backslash\left\{y_{1}, \ldots, y_{l}\right\}$ such that $f \simeq g$.

We close this section recalling a useful result proved in [1].

Theorem 2.3. Let $M^{m}$ be a compact differentiable manifold and let $k$ be an integer with $m \geq k \geq 2$. Then the relation $\varphi\left(M, \mathbf{R}^{k}\right)=\kappa_{1}$ is satisfied.

3. On the $\varphi$-category of the pairs $\left(G_{2, n}, \mathbf{R}^{m}\right)$ and $\left(G_{3, n}, \mathbf{R}^{m}\right)$

Theorem 3.1. (i) If the natural number $n$ is not a power of 2 , then we have 


$$
\varphi\left(G_{2, n}, \mathbf{R}^{m}\right)= \begin{cases}\geq 2^{p+1}-1 & \text { if } m=1 \text { and } n=2^{p}-1 \\ \aleph_{1} & \text { if } 2 \leq m \leq 2 n \\ \infty & \text { if } 2 n<m \leq 2 s-3 \\ ? & \text { if } 2 s-3<m<4 n-1 \\ 0 & \text { if } m \geq 4 n-1\end{cases}
$$

where $s=2^{r}$ is such that $2^{r-1} \leq n<2^{r}$.

(ii) If $n$ is a power of 2 , then we have

$$
\varphi\left(G_{2, n}, \mathbf{R}^{m}\right)= \begin{cases}N_{1} & \text { if } 2 \leq m \leq 2 n \\ \infty & \text { if } 2 n<m \leq 3 n-3 \\ ? & \text { if } 3 n-3<m<4 n-1 \\ 0 & \text { if } m \geq 4 n-1 .\end{cases}
$$

Proof. (i) The inequality $\varphi\left(G_{2,2 p-1}, \mathbf{R}\right) \geq 2^{p+1}-1$ follows from the inequality $\varphi(M, \mathbf{R}) \geq \operatorname{cat} M$ and from [2, Theorem 1.2]. The fact that $\varphi\left(G_{2, n}, \mathbf{R}^{m}\right)=\aleph_{1}$ for $2 \leq m \leq 2 n=\operatorname{dim} G_{2, n}$ follows from Theorem 2.3. For the proof of the fact that $\varphi\left(G_{2, n}, \mathbf{R}^{m}\right)=\infty$ under the conditions $2 n<m<2 s-3$, suppose that there exists a smooth mapping $f: G_{2, n} \rightarrow R^{2 s-3}$ with a finite number of critical points $x_{1}, x_{2}, \ldots, x_{l}$. Consider the usual embedding $i: G_{2, n-1} \hookrightarrow G_{2, n}$ and, according to Corollary 2.2, an immersion $g: G_{2, n-1} \rightarrow G_{2, n} \backslash\left\{x_{1}, \ldots, x_{l}\right\}$ homotopic to $i$. Then the application $f \circ g: G_{2, n-1} \rightarrow \mathbf{R}^{2 s-3}$ is an immersion, that is a contradiction with the fact that there is not any immersion from $G_{2, n-1}$ to $\mathbf{R}^{2 s-3}$ proved in [4, Theorem 1. (i)]. The fact that $\varphi\left(G_{2, n}, \mathbf{R}^{m}\right)=0$ for $m \geq 4 n-1$, follows from Whitney's embedding theorem.

The proof of the second statement can be made in an analogous manner, using the Corollary 2.2 and [4, Theorem 1. (ii)].

Theorem 3.2. Let $s=2^{r}$ be the natural number satisfying the condition $2^{r+1}<3 n<2^{r+2}$, with $n \geq 3$.

(i) If $\frac{2}{3}<n \leq s-3$, then we have

$$
\varphi\left(G_{3, n+1}, \mathbf{R}^{m}\right)= \begin{cases}\aleph_{1} & \text { if } 2 \leq m \leq 3 n+3 \\ \infty & \text { if } 3 n+3<m \leq 3 s-4 \\ ? & \text { if } 3 s-4<m<6 n+4 \\ 0 & \text { if } m \geq 6 n+5\end{cases}
$$

(ii) If $s \geq 8$, then we have 


$$
\varphi\left(G_{3, s-1}, \mathbf{R}^{m}\right)= \begin{cases}\aleph_{1} & \text { if } 2 \leq m \leq 3 s-3 \\ \infty & \text { if } 3 s-3<m \leq 4 s-4 \\ ? & \text { if } 4 s-4<m<6 s-7 \\ 0 & \text { if } m \geq 6 s-7\end{cases}
$$

and

$$
\varphi\left(G_{3, s}, \mathbf{R}^{m}\right)= \begin{cases}\aleph_{1} & \text { if } 2 \leq m \leq 3 s \\ \infty & \text { if } 3 s<m \leq 5 s-4 \\ ? & \text { if } 5 s-4<m<6 s-1 \\ 0 & \text { if } m \geq 6 s-1\end{cases}
$$

(iii) If $s<n<\frac{4}{3} s$, then we have

$$
\varphi\left(G_{3, n}, \mathbf{R}^{m}\right)= \begin{cases}\aleph_{1} & \text { if } 2 \leq m \leq 3 n \\ \infty & \text { if } 3 n<m \leq 6 s-4 \\ ? & \text { if } 6 s-4<m<6 n-1 \\ 0 & \text { if } m \geq 6 n-1\end{cases}
$$

Proof. (i) Theorem 2.3 ensures us that $\varphi\left(G_{3, n+1}, \mathbf{R}^{m}\right)=N_{1}$ if $2 \leq m \leq 3 n+3=$ $\operatorname{dim} G_{3, n+1}$, while $\varphi\left(G_{3, n+1}, \mathbf{R}^{m}\right)=0$ for $m \geq 6 n+5$, follows from Whitney's embedding theorem. It remains only to show that $\varphi\left(G_{3, n+1}, \mathbf{R}^{m}\right)=\infty$ for $3 n+3<m \leq 3 s-4$, that is, any differentiable mapping from $G_{3, n+1}$ to $\mathbf{R}^{3 s-4}$, has a finite number of critical points. Assume that there exists a mapping $f: G_{3, n+1} \rightarrow \mathbf{R}^{3 s-4}$ having a finite number of critical points $\left\{x_{1}, x_{2}, \ldots, x_{l}\right\}$ and consider the standard inclusion $j: G_{3, n} \hookrightarrow G_{3, n+1}$. Let $h: G_{3, n} \hookrightarrow G_{3, n+1} \backslash\left\{x_{1}, x_{2}, \ldots, x_{l}\right\}$ be the immersion (which is homotopic with $j$ ) ensured by the Corollary 2.2. Obviously $f \circ h: G_{3, n} \rightarrow \mathbf{R}^{3 s-4}$ is an immersion and we can consider the associated 3(s-n)-4-normal fibre bundle $v$. Taking into account the fact that $w_{3(s-n)-3}(v)=\bar{w}_{3(s-n)-3}\left(G_{3, n}\right)$, the relation $\bar{w}_{3(s-n)-3}\left(G_{3, n}\right) \neq 0$ proved in $[4$, Theorem 2 (i)] finishes the proof of the statement (i). The statements (ii) and (iii) can be proved analogously using the relations $\bar{w}_{s+3}\left(G_{3, s-2}\right) \neq 0, \bar{w}_{2 s}\left(G_{3, s-1}\right) \neq 0$ and $\bar{w}_{3(2 s-n+1)-3} \neq 0$ respectively, which are also proved in [4, Theorem 2 (ii)] and [4, Theorem 2 (iii)] respectively.

\section{On the $\varphi$-category of the pair $\left(P_{n}(\mathbf{R}), \mathbf{R}^{m}\right)$}

In this section the case of the pair $\left(P_{n}(\mathbf{R}), \mathbf{R}^{m}\right)$ will be treated. For this purpose we need some helpful results. 
Lemma 4.1. If $A \subseteq S^{n},(n \geq 2)$ is a finite set, then there exists $x \in S^{n}$ such that $(x)^{\perp} \cap A=\emptyset$ where $\langle x\rangle^{\perp}$ denotes the orthogonal complement of $x$ with respect to the usual scalar product from $\mathbf{R}^{n+1}$.

Proof. The proof will be made by induction with respect to $k=|A|$. If $k=1$, then $A=\{a\}$ and we can choose $x=a$. Suppose that $|A|=k+1$ and choose $a \in A$. From the induction hypothesis it follows that there exists $x^{\prime} \in S^{n}$ such that $\left\langle x^{\prime}\right\rangle^{\perp} \cap(A \backslash\{a\})=\emptyset$. If $a \notin\left\langle x^{\prime}\right)^{\perp}$ choose $x=x^{\prime}$, else we choose $\theta \in(0, m)$ where

$$
m=\min \left\{\left|\operatorname{arctg} \frac{\left\langle a, x^{\prime}\right\rangle}{\left\langle a, a^{\prime}\right\rangle}\right|: a^{\prime} \in A \backslash\{a\}\right\},
$$

with $m=\frac{\pi}{2} \quad$ if $\quad\left\langle a, a^{\prime}\right\rangle=0(\forall) a^{\prime} \in A \backslash\{a\}$, and $x=\cos \theta x^{\prime}+\sin \theta a \in S^{n}$. Obviously $\langle a, x\rangle=\sin \theta>0$, that is $a \notin\langle x\rangle^{\perp}$ and since $\left\langle a^{\prime}, x\right\rangle=\cos \theta\left\langle a^{\prime}, x^{\prime}\right\rangle+\sin \theta\left\langle a, a^{\prime}\right\rangle \neq$ $0\left(\forall a^{\prime} \in A \backslash\{a\}\right.$, it implies that $(A \backslash\{a\}) \cap\langle x\rangle^{\perp}=\emptyset$ which together with $a \notin\langle x\rangle^{\perp}$ leads to the conclusion that $\langle x\rangle^{\perp} \cap A=\emptyset$.

Proposition 4.2. If $A \subseteq S^{n}, n \geq 2$ is a finite set $\mathbf{Z}_{2}$-invariant (symmetric), then there exists a $\mathbf{Z}_{2}$-equivariant (odd) embedding $f: S^{n-1} \rightarrow S^{n} \backslash A$.

Proof. Let us consider $x \in S^{n}$ such that $\langle x\rangle^{\perp} \cap A=\emptyset$. Because the orthogonal group $O(n)$ acts transitively on $S^{n}$, it follows that there exists $T \in O(n)$ such that $T\left(e_{n+1}\right)=x$ where $e_{n+1}=(0, \ldots, 0,1) \in \mathbf{R}^{n+1}$. But since $\left\langle e_{n+1}\right\rangle^{\perp}=\left\{\left(x_{1}, x_{2}, \ldots, x_{n+1}\right) \in \mathbf{R}^{n+1} \mid x_{n+1}=\right.$ $0\} \simeq \mathbf{R}^{n}$ and $T$ is an orthogonal diffeomorphism which leaves invariant the sphere $S^{n}$, it implies that $T\left(\mathbf{R}^{n}\right)=\langle x\rangle^{\perp}$. Choose $f=\left.T\right|_{s^{n-1}}$.

Corollary 4.3. If $A \subseteq P_{n}\left(\mathbf{R}^{n}\right),(n \geq 2)$ is a finite subset, then there exists an immersion $g: P_{n-1}(\mathbf{R}) \rightarrow P_{n}(\mathbf{R}) \backslash A$.

Proof. Let $f: S^{n-1} \rightarrow S^{n} \backslash p_{n}^{-1}(A)$, where $p_{n}: S^{n} \rightarrow P_{n}(\mathbf{R})$ is the canonical projection, be the embedding ensured by Proposition 4.2. $g$ will be chosen as being the mapping which makes commutative the following diagram:

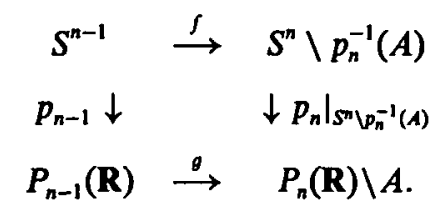

Let $A$ be a finite subset of $P_{n}(\mathbf{R})$ and $E\left(\gamma_{n}^{l}(A)\right)$ be the subset of $\left(P_{n}(\mathbf{R}) \backslash A\right) \times \mathbf{R}^{n+1}$ consisting in all pairs $(\{ \pm x\}, v)$ such that $v$ is a multiple of $x$. Define $\pi_{A}: E\left(\gamma_{n}^{1}(A)\right) \rightarrow$ $P_{n}(\mathbf{R}) \backslash A$ by $\pi_{A}(\{ \pm x\}, v)=\{ \pm x\}$. Hence every fibre $\pi_{A}^{-1}(\{ \pm x\})$ can be identified with the straight line through $x$ and $-x$ from $\mathbf{R}^{n+1}$. The resultant fibre bundle $\gamma_{n}^{\prime}(A)$ will be 
called the canonical line bundle over $P_{n}(\mathbf{R}) \backslash A$. Note that $\gamma_{n}^{1}(\emptyset)$ is even the canonical line bundle $\gamma_{n}^{1}$ (over $P_{n}(\mathbf{R})$ ) defined in [3, pp. 16].

Proposition 4.4. The total Stiefel-Whitney class of the canonical line fibre bundle $\gamma_{n}^{1}(A)$ over $P_{n}(\mathbf{R}) \backslash A$ is given by

$$
\omega\left(\gamma_{n}^{1}(A)\right)=1+a_{A}
$$

where $a_{A} \in H^{\prime}\left(P_{n}(\mathbf{R}) \backslash A ; \mathbf{Z}_{2}\right)$ is not zero.

Proof. Let $j^{\prime}: S^{1} \rightarrow S^{n} \backslash p_{n}^{-1}(A)$ be a $Z_{2}$-equivariant embedding. Obviously $j^{\prime}$ induces an immersion $j: P_{1}(\mathbf{R}) \rightarrow P_{n}(\mathbf{R}) \backslash A$ covered by an application of fibrations from $\gamma_{1}^{1}$ to $\gamma_{n}^{1}(A)$. Therefore denoting by $a_{A}$ the Stiefel-Whitney class $\omega_{1}\left(\gamma_{1}(A)\right)$, one can say that $j^{*}\left(a_{A}\right)=\omega_{1}\left(\gamma_{1}^{1}\right) \neq 0$ which shows that $a_{A} \neq 0$.

Remark. If $n \geq 2$, then $a_{A}^{k} \neq 0$, ( $\left.\forall\right) k \in\{1,2, \ldots, n-1\}$. Indeed if $k: P_{1}(\mathbf{R}) \rightarrow P_{n-1}$ (R) denotes the usual inclusion, which can be obviously covered by an application of fibrations from $\gamma_{1}^{1}$ to $\gamma_{n-1}^{1}$ and $j: P_{n-1}(\mathbf{R}) \rightarrow P_{n}(\mathbf{R}) \backslash A$ the immersion ensured by Corollary 4.3 , which can be also covered by an application of fibrations from $\gamma_{n-1}^{1}$ to $\gamma_{n}^{1}(A)$, then from the second axiom of the Stiefel-Whitney classes, it follows that $k^{*}\left(j^{*}\left(a_{A}\right)\right)=\omega_{1}\left(\gamma_{1}^{1}\right) \neq 0$, and therefore $j^{*}\left(a_{A}\right)=a \in H^{1}\left(P_{n-1}(\mathbf{R}) ; \mathbf{Z}_{2}\right)$ is the generator (obviously non zero) of $H^{1}\left(P_{n-1}(\mathbf{R}) ; \mathbf{Z}_{2}\right)$. But since $a^{k}=j^{*}\left(a_{A}^{k}\right)$ is the generator (obviously non zero) of $H^{k}\left(P_{n-1}(\mathbf{R}) ; \mathbf{Z}_{2}\right)$ for any $k \in\{1,2, \ldots, n-1\}$, it implies that $a_{A}^{k} \neq 0$, for each $k \in\{1,2, \ldots, n-1\}$.

Using a similar judgement with that from [3, Theorem 4.5, p. 45] one can show that the manifold $P_{n}(\mathbf{R}) \backslash A$ has the total Stiefel-Whitney class

$$
\omega\left(P_{n}(\mathbf{R}) \backslash A\right)=\left(1+a_{A}\right)^{n+1}=1+\left(\begin{array}{c}
n+1 \\
1
\end{array}\right) a_{A}+\left(\begin{array}{c}
n+1 \\
2
\end{array}\right) a_{A}^{2}+\cdots+\left(\begin{array}{c}
n+1 \\
n
\end{array}\right) a_{A}^{n} .
$$

For $n=2^{r}$ we get

$$
\omega\left(P_{2^{r}}(\mathbf{R}) \backslash A\right)=\left(1+a_{A}\right)^{2^{r}+1}=1+a_{A}+a_{A}^{2 r}
$$

and also

$$
\bar{\omega}\left(P_{2^{r}}(\mathbf{R}) \backslash A\right)=1+a_{A}+a_{A}^{2}+\cdots+a_{A}^{2^{\prime}-1} .
$$

Theorem 4.5. If $n$ is a natural number such that $n+1$ and $n+2$ are not powers of 2 , then the $\varphi$-category of the pair $\left(P_{n}(\mathbf{R}), \mathbf{R}^{m}\right)$ is given by: 


$$
\varphi\left(P_{n}(\mathbf{R}), \mathbf{R}^{m}\right)= \begin{cases}n+1 & \text { if } m=1 \\ \aleph_{1} & \text { if } 2 \leq m \leq n \\ \infty & \text { if } n<m \leq 2^{\left[\log _{2} n\right]+1}-2 \\ ? & \text { if } 2^{\left[\log _{2}\right]+1}-1 \leq m \leq 2 n-2 \\ 0 & \text { if } m \geq 2 n-1\end{cases}
$$

Proof. The case $m=1$ is justified in [6]. The fact that $\varphi\left(P_{n}(\mathbf{R}), \mathbf{R}^{m}\right)=\kappa_{1}$ for $2 \leq m \leq n$ follows from Theorem 2.3. Consider firstly the case when $n$ is a power of 2 , that is, $n=2^{\left[\log _{2} n\right]}$. Assume that $n<m \leq 2^{\left[\log _{2} n\right]+1}-2$ and that there exists $f: P_{2^{\left(\log _{2} n\right)}}(\mathbf{R}) \rightarrow \mathbf{R}^{m}$ such that $C(f)$ is finite. If $v$ is the associated normal fibre bundle (over $P_{2^{\left[\log _{2} n\right]}}(\mathbf{R}) \backslash C(f)$ ) to the immersion $\left.f\right|_{P_{\left.2^{\left[\left(\log _{2}\right)\right.}\right)}(\mathbf{R}) \backslash(U)}$ then

$$
\omega(v)=\bar{\omega}\left(P_{2^{\left[l o g_{2} n\right]}}(\mathbf{R}) \backslash C(f)\right)=1+a_{C(n)}+a_{c(n)}^{2}+\cdots+a_{C(n)}^{2^{\left[l_{0} n\right]-1}} .
$$

But since $v$ is a $m-2^{\left[\log _{2} n\right]}$-vector fibre bundle and $a_{C(n)}^{2^{\left[\log _{2} n\right]-1}} \neq 0$ it follows that $m-2^{\left[\log _{2} n\right]} \geq 2^{\left[\log _{2} n\right]}-1$ which means that $m \geq 2^{\left[\log _{2} n\right]+1}-1>2^{\left[\log _{2} n\right]+1}-2$ that is a contradiction. If $n$ is not a power of 2 , then the hypothesis of the theorem ensures that $2^{\left[\log _{2} n\right]}+1 \leq n \leq 2^{\left[\log _{2} n\right]+1}-3$. Assume that $n<m \leq 2^{\left[\log _{2} n\right]+1}-2$ and that there exists a differentiable application $g: P_{n}(\mathbf{R}) \rightarrow \mathbf{R}^{m}$ such that $C(g)$ is finite. If $h: P_{2^{\left(\log _{2 n} n\right.}}(\mathbf{R}) \rightarrow P_{n}(\mathbf{R}) \backslash C(g)$ is the immersion ensured by Corollary 4.3 , then obviously $g \circ h: P_{2^{\left[\log _{2} n\right.}}(\mathbf{R}) \rightarrow \mathbf{R}^{m}$ is an immersion. If $v^{\prime}$ is the associated normal fibre bundle (over $\left.P_{2^{\left[\log _{2} n\right)}}(\mathbf{R})\right)$ of the immersion $g \circ h$, then $w\left(v^{\prime}\right)=\bar{w}\left(P_{2^{\left(1002^{n}\right)}}(\mathbf{R})\right)=1+a+a^{2}+\cdots+a^{2^{\left(\log _{2} n\right)}-1}$. But since $v^{\prime}$ is a $m-2^{2^{\left[\log _{2} n\right]}}$-vector fibre bundle and $a^{2^{\left[\log _{2} n\right]}-1} \neq 0$ it follows that $m-2^{\left[\log _{2} n\right]} \geq 2^{\left[\log _{2} n\right]}-1$ which means that $m \geq 2^{\left[\log _{2} n\right]+1}-1>2^{\left[\log _{2} n\right]+1}-2$ that is a contradiction. The fact that $\varphi\left(P_{n}(\mathbf{R}), \mathbf{R}^{m}\right)=0$ for $m \geq 2 n-1$ follows from Whitney's embedding theorem.

Corollary 4.6. If $m$ and $n$ are natural numbers such that $n+1$ and $n+2$ are not powers of 2 and $2 \leq m \leq 2^{2^{\left(\operatorname{loo}_{2} n\right)+1}}-2$, then any smooth $\mathbf{Z}_{2}$-invariant (even) mapping $f: S^{n} \rightarrow \mathbf{R}^{m}$ has an infinite number of critical orbits, that is, there exists infinitely many points $x \in S^{n}$ such that $x$ and $-x$ are critical points of $f$.

\section{REFERENCES}

1. D. Andrica and C. Pintea, Critical points of vector-valued functions (Proceedings of the 24th National Conference of Geometry and Topology, July 5-9, 1994, Romania, University of Timişoara, 1996).

2. I. BERSTEIN, On the Lusternik-Schnirelmann category of Grassmannians, Math. Proc. Cambridge Philos. Soc. 79 (1976), 129-134.

3. J. W. Milnor and J. D. StashefF, Characteristic classes (Princeton, New Jersey, 1974). 
4. V. OPRoIU, Some non-embedding theorems for the Grassmann manifolds, Proc. Edinburgh Math. Soc. 20 (1976-77), 177-185.

5. R. S. Palais and C. L. Terng, Critical Point Theory and Submanifold Geometry (SpringerVerlag, Lecture Notes in Mathematics, 1988).

6. F. TAkens, The minimal number of critical points of a function on a compact manifold and the Lusternik-Schnirelmann Category, Invent. Math. 6 (1968), 197-244.

"BABEŞ-BOLYAI" UNIVERSITY

DEPARTMENT OF MATHEMATICS

STR. Kogălniceanu 1

3400 Cluj-Napoca

ROMANIA

E-mail: cpintea@math.ubbcluj.ro 